\title{
Calibration and Reference Samples in Trace Metals Determination in Serum by Graphite Furnace Atomic Absorption Spectrometry
}

\author{
Adam Hulanicki*, Ewa Bulska* and Katarzyna Wrobel** \\ *Department of Chemistry, University of Warsaw, Pasteura 1, 02-093 Warsaw, Poland \\ **Institute of Chemistry, University of Warsaw-Branch in Bialystok, al. J. Pilsudskiego, Biayłstok, Poland
}

\begin{abstract}
Determination of trace metals in blood, serum and plasma presents an important problem of increasing importance from both medical and environmental points of view. For many metals, atomic absorption spectrometry is a method of choice. Nevertheless, a significant obstacle arises because of the lack of availability of proper reference materials, especially ones having the metal content of the order of a few nanograms per milliliter. In our studies dealing with determination of lead, cadmium, cobalt, manganese, aluminium and chromium, calibration with model aqueous solutions and with serum simulating albumin solution was used and the effects of matrix materials were noted. Some improvement may be achieved when matrix modifiers-stabilizers have been used. The often used quantification procedure based on standard addition may also lead to erroneous results because the form in which the analyte is added to the sample does not reflect the speciation in the real samples. This indicates that the problems cannot be considered as completely solved and further studies are indispensable.
\end{abstract}

Keywords Atomic absorption spectrometry, serum, trace metal determination, calibration, reference material

Nearly 60 years ago, an American analyst, G.E.F. Lundell, wrote that "the determinator's salvation lies in development of truly selective methods of analysis, and his final resting place will be a heaven in which he has a shelf containing 92 reagents, one for each element...". 1 The conditions and possibilities changed with the development of modern analytical techniques, but in spite of the time lapse the dreams remained and we are still far from the truly selective, or as we would prefer to say-truly specific methods.

A truly specific method should mean that the analytical signal, which forms the basis of the quantitative evaluation of the element content, is independent from any concomitant materials present in the sample. Thus the lack of influence of the matrix components means that the magnitude of the signal obtained for each model, usually an aqueous standard solution, of a given analyte may serve as a measure of its content in any other sample of arbitrarily chosen complexity of composition. This also means that the analytical procedure is interference-free and the signal depends exclusively on the nature of the analyte, on its concentration and in most instances on the instrumental parameters and experimental conditions, the last two being usually kept constant. There have been several attempts to find conditions in which atomic absorption spectrometry could be regarded as an absolute method of analysis. Such an approach was initiated by L'vovi ${ }^{2-4}$

This paper was presented at the Kitami Conference of ICAS '91. and continued by other scientists, as for example by Slavin and Manning 5,6 , who have introduced the Stabilized Temperature Platform Furnace concept.

The experimental data and their comparison with the theoretically calculated values indicate that the ratio of theoretical to experimental characteristic masses in many cases differ from one and may vary from 0.09 for barium to 1.69 for lead (Fig. 1). Therefore the best experimental conditions, e.g., the temperatures, matrix modifiers, kind and pretreatment of the sample carrier cannot in

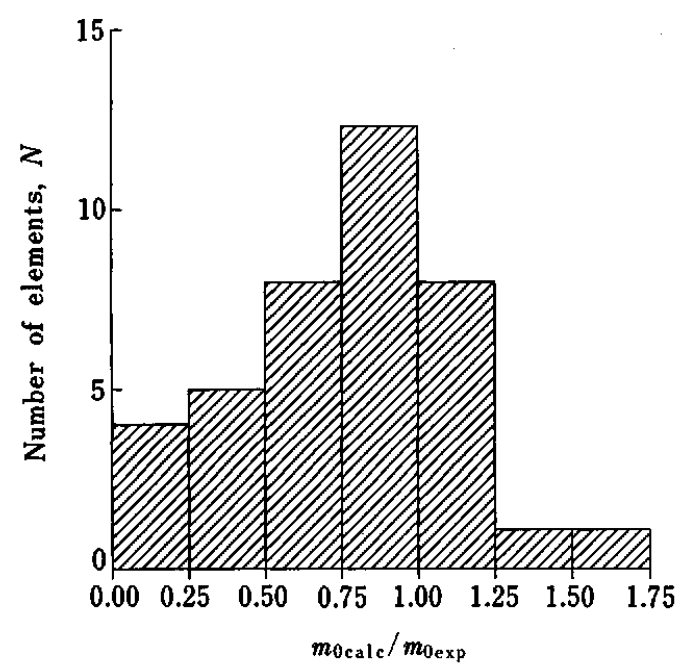

Fig. 1 The ratio of calculated to experimental values of characteristic masses, $m_{0}$, for 39 elements. ${ }^{4}$ 
Table 1 Serum Certified Reference Materials

\begin{tabular}{|c|c|c|c|c|c|c|}
\hline & \multicolumn{6}{|c|}{ Trace metals, ppb } \\
\hline & $\mathrm{Al}$ & $\mathrm{Cr}$ & Mn & $\mathrm{Co}$ & $\mathrm{Cd}$ & $\mathbf{P b}$ \\
\hline Reference values ${ }^{8}$ & $<10$ & $0.1-0.2$ & 0.5 & $0.1-0.2$ & $0.1-0.2$ & 1 \\
\hline Bovine Serum SRM 8491 & 13 & 0.30 & $2.6^{\circ}$ & 1.2 & - & - \\
\hline Bovine Serum SRM 1598 & 3.7 & 0.14 & 3.8 & 1.2 & 0.09 & - \\
\hline Human Serum ${ }^{7}$ & 20.2 & 0.76 & 7.7 & 3.6 & 2.0 & - \\
\hline Human Serum SRM 909 & - & 108 & - & - & 1.46 & 2.4 \\
\hline Blood BCR 194, 195, 196 & - & - & - & - & $0.5-12.4$ & $126-770$ \\
\hline
\end{tabular}

general be predicted theoretically and are in the majority of analytical procedures chosen by trial and error. To elucidate and in consequence to suppress the side reactions such as e.g., formation of gaseous carbides, cyanides etc. still more efforts are needed. At present at least, there are numerous "difficult" elements for which the theoretical calculations cannot give even semiquantitative evaluation.

On this basis, we can state that we are still far from the absolute analysis, which is at present not possible, especially for complex materials with unknown matrices. To those materials belong often biological samples such as tissues and fluids. To obtain reliable and accurate analytical results good Standard Reference Materials (SRM) are needed.

The ultimate conditions for SRM are:

i) the level of concentration of the analytes should be as close as possible to their levels in analyzed samples;

ii) the concentrations of the analytes must be known with the highest possible accuracy;

iii) the matrix should resemble, or better, be identical with the matrix of the analyzed samples.

Those conditions are difficult to be fulfilled when extremely low concentrations are determined and when the matrix is not indefinitely stable in time, when it is not perfectly homogeneous, or when it exerts a significant effect not only on the proper signal but also on the background.

The first generation of SRM for determination of trace concentrations in blood or serum was generally characterized by higher content of trace elements compared to real samples; homogeneity was not ideal but the matrix composition was adequate. The second generation $^{7}$ of SRM has lower contents of many trace metals, which roughly correspond to those of the references. On the other hand it must also be taken into account that the reference values are not always constant. Very low reference values for many metals have been given by Versieck and Cornelis. ${ }^{8}$ Depending however on the individual pathology and the background, which includes diet, environmental conditions, working place etc. they may vary even by one order of magnitude, as has been recently shown by some investigations. ${ }^{9}$ Such SRM are not easily available; they are expensive and have not been certified for all elements of interest (Table 1).
In our studies we decided to approach the accurate results of diagnostic value by depending on our own analytical samples and procedures. The complete procedure incorporates sample, i.e., serum, pretreatment, including the addition of the matrix modifierstabilizer, the choice of optimal temperature programmes for electrothermal atomic absorption and finally the evaluation of analytical signals.

In treatment of standards and samples, the freezedrying is often considered as an important step. It enables storage of samples and standards at room temperature, and ensures better long term stability and homogeneity. Taking into account all advantages and disadvantages (possible loss of some analytes, difficulties in using standard addition techniques and in carrying out speciation analysis), the answer is rather positive for the freeze-drying procedures, especially when better precision is expected. There is substantial evidence that freeze-drying improves precision of determination (Table 2).

In determination of trace metals in blood samples the

Table 2 Homogeneity of reconstituted serum samples expressed as imprecision (RSD, $\% ; n=5$ )

\begin{tabular}{cccc}
\hline Pretreatment & Element & Fresh & Freeze-dried \\
\hline $\mathrm{H}_{2} \mathrm{O}$ dilution & $\mathrm{Mn}$ & 9.0 & 8.1 \\
& $\mathrm{Cr}$ & 29 & 10 \\
0.1 mol $/ 1 \mathrm{HNO}_{3}$ & $\mathrm{Cd}$ & 7.1 & 6.0 \\
dilution & $\mathrm{Mn}$ & 13 & 10 \\
& $\mathrm{Cr}$ & 1.7 & 1.4 \\
& $\mathrm{Cd}$ & 6.0 & 5.5 \\
& $\mathrm{~Pb}$ & 13 & 5.8 \\
& $\mathrm{Co}$ & 3.8 & 3.2 \\
$0.1 \%$ Triton $\mathrm{X}^{-100}$ & $\mathrm{Al}$ & 5.9 & 3.0 \\
dilution & $\mathrm{Cr}$ & 8.5 & 1.6 \\
& $\mathrm{Co}$ & 8.8 & 6.6 \\
$\mathrm{HNO}_{3}+\mathrm{Mg}\left(\mathrm{NO}_{3}\right)_{2}$ & $\mathrm{Al}$ & 5.9 & 2.5 \\
dilution & $\mathrm{Al}$ & 8.0 & 2.0 \\
$\mathrm{HNO}_{3}+\mathrm{H}_{3} \mathrm{PO}_{4}$ & & & \\
dilution & $\mathrm{Cd}$ & 5.3 & 4.9 \\
$\mathrm{Pd}_{\text {as modifier, }} \mathrm{HNO}_{3}$ & $\mathrm{~Pb}$ & 6.0 & 4.7 \\
dilution & $\mathrm{Co}$ & 5.3 & 2.3 \\
\hline
\end{tabular}


most commonly used sample material is serum, which is obtained by elimination of cellular components and fibrin from whole blood. Another material for analysis is plasma, which is obtained by heparin sampling and subsequent centrifugation. Both these fractions can be analyzed by AAS without complete mineralization. Mineralization, e.g., in a Teflon bomb is a timeconsuming procedure and is also prone to contamination. But in the case of whole blood, the large amount of organic matter makes mineralizatin unavoidable.

The protein content of serum and plasma influences the analyte behavior during atomization and makes impossible the use of aqueous solution calibration. This can be illustrated from the determination of manganese. ${ }^{11}$ The aqueous standards without or with dilute nitric acid show the maximum on th time profile at $1600 \mathrm{~K}$. A similar profile was obtained when serum containing manganese was diluted with water or when manganese was determined in sample containing albumin and nitric acid. However, when serum was diluted with $0.1 \mathrm{~mol} \mathrm{l}^{-1}$ nitric acid, the peak was shifted to $1800 \mathrm{~K}$.

Such behavior in serum samples was attributed to the synergic effect of calcium and phosphate present in serum, which also act similarly when purposely added to

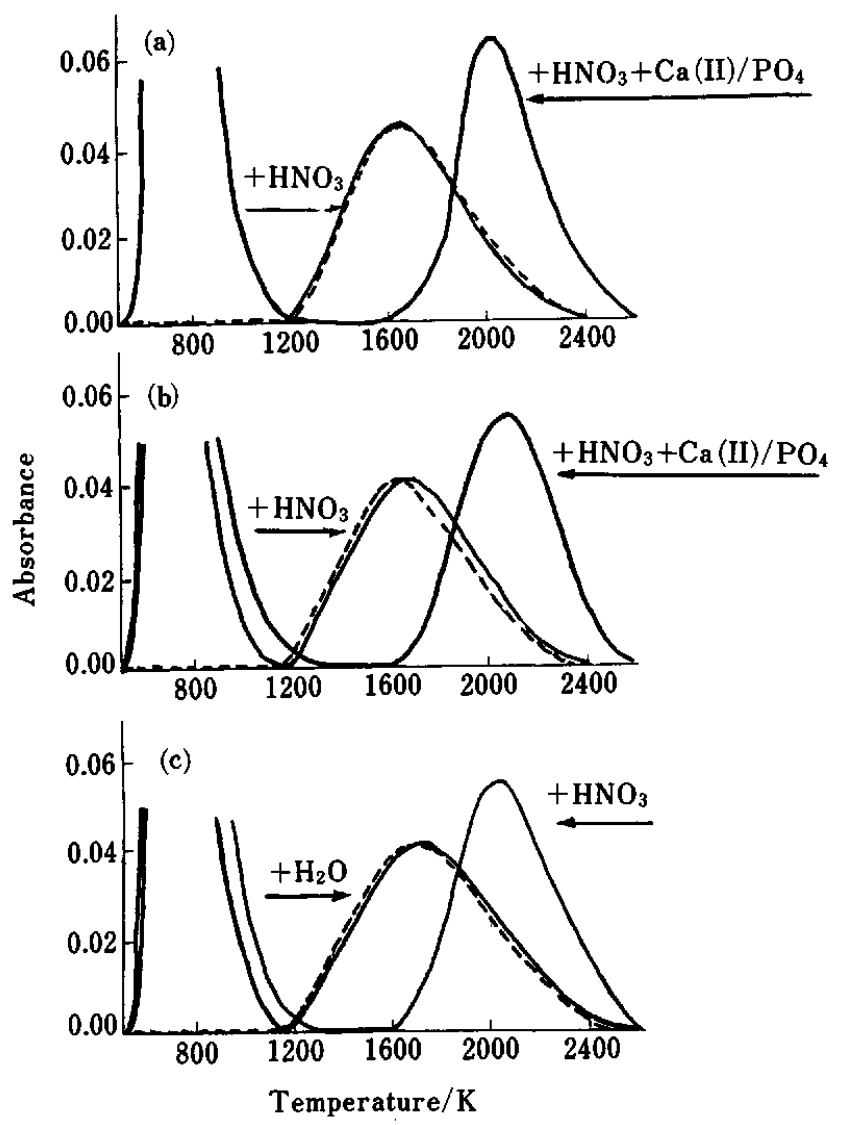

Fig. 2 Time profiles for $5 \mathrm{ng}$ of manganese in aqueous solutions (a), in 5\% albumin solutions (b) and in serum sample solutions (c). The dashed line stands for aqueous Mn standard. The additions are indicated by solid lines. ${ }^{10}$ an aqueous solution of manganese ${ }^{11}$ (Fig. 2). In such a sample, a matrix modifier-stabilizer is formed from components of serum, but this is not observed when pure albumin is taken as a substitute for serum. Therefore, albumin solutions cannot mimic serum in calibration procedures. The inspection of the ashing curves confirms this statement and gives an indication of what solution composition should be used for calibration. To obtain consistent results with model solutions, the serum samples have to be diluted with nitric acid and a calcium-phosphate modifier must be added. On the other hand, the standards for calibration could be prepared on the basis of a commercial preparationHumanalbin ${ }^{12}$-used for infusions. This solution contains human proteins and some accompanying electrolytes. Similar behavior was found in the case of cobalt determination. ${ }^{12}$

In the case of another element of interest-lead-the effect of proteins was also observed on time profiles and ashing curves. Lead in the presence of albumin or in serum cannot be determined because of a large nonspecific background which merges with the element signal. When phosphoric acid is added, the nonspecific signal is separated from the lead signal and the determination is possible in the presence or absence of albumin. In this case, phosphoric acid ${ }^{13}$ was used as the matrix modifier-stabilizer and, for standards, humanalbin preparation was used. A similar procedure with the same modifier ${ }^{13}$ was used for determination of cadmium.

Among elements of special interest is aluminum. In

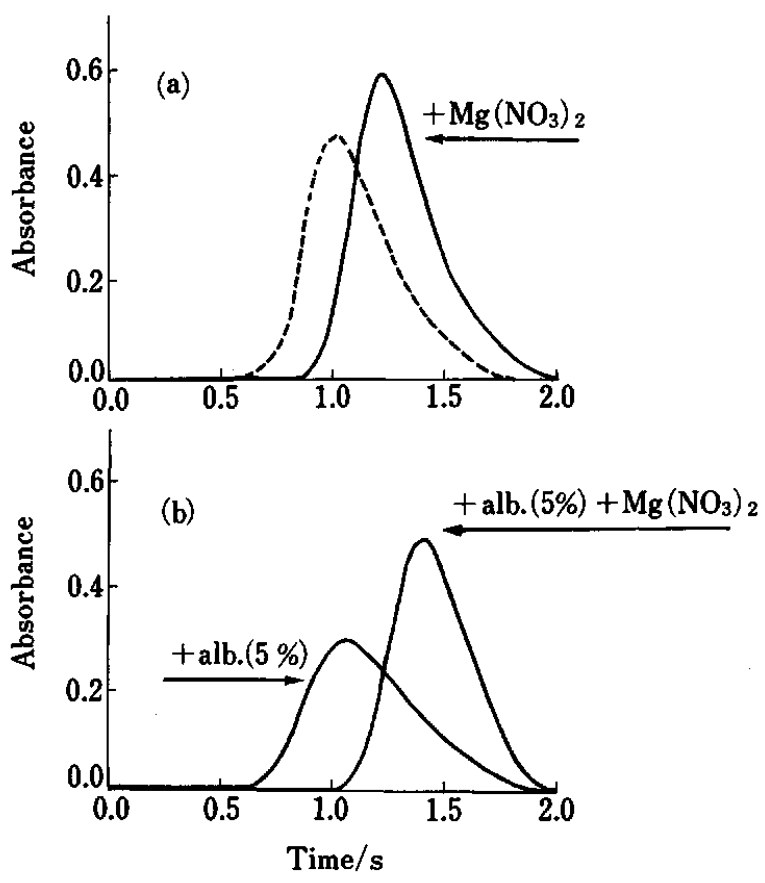

Fig. 3 Time profiles for $0.05 \mathrm{ng}$ of aluminium in aqueous (a) and in 5\% albumin solution (b). Dashed line stands for aqueous $\mathrm{Al}$ standard. The additions are indicated by solid lines. 
the presence of proteins, the analytical signal decreases and the ashing temperature cannot be raised above $1500 \mathrm{~K}$. When magnesium nitrate ${ }^{14}$ was added as a modifier-stabilizer then ashing till $1770 \mathrm{~K}$ is possible and the sensitivity also increases (Fig. 3). The same modifier was used for determination of chromium. ${ }^{15}$ Another choice is palladium, widely used as a modifier. ${ }^{16}$ For all studied elements, the addition of the modifier is also advantageous because of increase of sensitivity.

In determination of all these elements, several sample pretreatment procedures were tested. Among them were: ${ }^{11}$ dilution with water, with acids or with surfactant solutions, deproteination, wet and dry mineralization. The choice of the best procedure was based on several criteria. ${ }^{16}$ Most important were:

i) shape and position of the signal on time axis,

ii) scatter of results within each procedure,

iii) scatter of results between different procedures,

iv) difference of results between calibration graph and standard addition techniques,

v) simplicity, time and labor consumption, danger of contamination.

The choice of the optimal procedure was of special importance when various fractions of blood were analyzed for cobalt content. ${ }^{12}$ In this case, only three main procedures of sample pretreatment were compared: dilution with water, dilution with $0.1 \mathrm{~mol} / 1$ nitric acid and digestion with concentrated nitric acid in PTFE bomb. These tests have also confirmed that for serum the dilution with nitric acid is the best procedure. This treatment was, however, unsuitable for plasma analysis because after addition of acid the solution became turbid and a precipitate appears. This caused inhomogeneity of the sample and a poor precision. For these samples the best results were obtained with simple 4-fold dilution with water.

Both dilution procedures were found unsuccessful for analysis of whole blood because large amounts of proteins make the direct determination practically impossible. The scatter of results exceeded 50\%, compared with $30 \%$ which was found for mineralized samples. In all experiments the samples were freezedried. Comparison of final results indicates, however, that our procedures are still not perfect and our knowledge about trace metal distribution among the blood fractions is insufficient. For such problems, the appropriate SRM would be of significant help, but it will be not easy to prepare such materials.

The important problem of calibration is connected with use as a measure of concentration either integral absorbance or peak height. The STPF concept as well as the absolute method approach requires measurement of integrated absorbance. Such measurement at least in principle should make the result independent from the atomization kinetics and in consequence from the shape of the signal profile.

When analyzed samples are rich in nonvolatile organic matter, some carbonized residue may remain in the graphite furnace. This results in obtaining a tailing analytical signal, increased background and in addition some memory effects. In consequence, it is difficult to give reasonable integration limits and best conditions for elimination of the background. The peak height is less influenced by these factors and the results are more reliable, in particular when matrix modifier-stabilizer is added. In principle, the modifier should unify the atomization mechanisms which is also accompanied by the increase of the peak height. Such improvement of sensitivity is not observed when integral absorbance is taken as the basis of quantitation. Nevertheless, it must be remembered that such effects are observed to various degrees for different elements, e.g., they are significant for cadmium but small for aluminum. In spite of that, for determination of all elements the peak height was accepted as the measure of element quantity.

Comparison of results obtained by two principal techniques: calibration graph and standard addition, shows that the results may be statistically different. Nearly as a rule it was found that results from standard addition are higher by 5 to $10 \%$ (Table 3). Only in the case of manganese has no such difference been observed. There is not enough experimental evidence to explain this. It may be supposed that addition of analyte ions to the sample solution is made in the form of free ions, whereas in the sample the analyte occurs at least partly in the form of organic complexes. Because of the inertness of the species, they do not reach true equilibrium. On the other hand, it is known that analytes differently bound with ligands may undergo different atomization paths. This is known particularly for chelates with different donor atoms. ${ }^{17}$ In all studied instances some time was left for equilibration, but whether it has been reached was not checked.

The purpose of this study was not only to investigate the trace metal contents in serum for themselves, but first of all to reach a value of diagnostic importance in medicine. Such important results were obtained e.g., for aluminum. ${ }^{15}$ It was found that the mean content for patients exposed to excessive aluminum pollution, living close to the foundry and patients after oral administration of aluminum-containing drugs was nearly two

Table 3 Differences among results obtained by various calibration techniques for aluminum and cadmium determination in serum after dilution with $0.1 \mathrm{~mol} / 1 \mathrm{HNO}_{3}$

\begin{tabular}{llllll}
\hline & \multicolumn{2}{c}{ Calibration graph } & \multicolumn{2}{c}{ Standard addition } \\
\cline { 2 - 3 } \cline { 5 - 6 } & Cd & \multicolumn{2}{c}{$\begin{array}{c}\text { Al } \\
\text { Peak }\end{array}$} & $\begin{array}{c}\text { Cd } \\
\text { height }\end{array}$ & $\mathrm{Al}$ \\
Mean value, ppb & 1.04 & 4.56 & 1.10 & 4.77 \\
SD, ppb & 0.06 & 0.11 & & 0.10 & 0.27 \\
RSD, \% & 5.3 & 2.5 & 9.1 & 5.6 \\
& \multicolumn{4}{c}{ Integral absorbance } \\
Mean value, ppb & 1.08 & 4.90 & 1.11 & 4.93 \\
SD, ppb & 0.07 & 0.26 & 0.16 & 0.41 \\
RSD, \% & 6.8 & 5.3 & 14.0 & 8.3 \\
\hline
\end{tabular}


times higher than for the control group. It was also tested that the level of aluminum in serum rapidly, i.e., during $3 \mathrm{~h}$, decreases to that found for the control group.

These results indicate that meaningful diagnostic analytical results can be obtained using properly chosen conditions and a thoroughly tested calibration technique even without SRM. It is evident that the levels of trace elements are higher in our experiments than the reference values given by Versieck and Cornelis. ${ }^{8}$ It must be remembered, however, that serum was taken from patients living in highly polluted areas who had been hospitalized for diseases which may be accompanied or caused by excessive levels of trace metals. Nevertheless the SRM when available may significantly decrease the time and labour consumption necessary for obtaining reasonable and useful data.

Those considerations refer only to the determination of total metal content. The next step should be the speciation analysis of trace metals in biological fluids. Determination of individual species presents obviously many more difficulties and for that purpose a new, third generation of SRM will be needed. The level of concentration of species is lower than for the total content. This is a new exciting challenge for analysts. Through new Standard Reference Materials and through attempts to develop absolute methods of analysis they will hopefully come nearer to the discovery of the secrets of life.

\section{References}

1. G. E. F. Lundell, Ind. Eng. Chem., (Anal. Ed.), 5, 1 (1933).

2. B. V. L'vov, Spectrochim. Acta, 33B, 153 (1978).
3. B. V. L'vov, Spectrochim. Acta, 39B, 149 (1984).

4. B. V. L'vov, V. G. Nikolaev, E. A. Norman, L. K. Polzik and M. Mojica, Spectrochim. Acta, 41B, 1043 (1986).

5. W. Slavin and D. C. Manning, Anal. Chem., 51, 261 (1979).

6. Z. Grobenski and B. Welz, Chem. Anal. [Warsaw], 35, 141 (1990).

7. J. Versieck, L. Vanballenberghe, A. de Kesel, J. Hoste, B. Wallaeys, J. Vandenhaute, N. Baeck, H. Steyaert, A. Byrne and F. W. Sunderman Jr., Anal. Chim. Acta, 204, 63 (1988).

8. J. Versieck and R. Cornelis, "Trace Elements in Human Plasma or Serum", CRC Press, Boca Raton, 1989.

9. J. M. Christensen, S. Mikkelsen and A. Skov, "Chemical Toxicology and Clinical Chemistry of Metals", ed. S. S. Brown and J. Savory, pp. $65-68$, Academic Press, London, 1983.

10. H. T. Delves, Clin Endocrinol. Metabol., 14, 725 (1985).

11. A. Hulanicki, E. Bulska, B. Godlewska and K. Wróbel, Anal. Lett., 22, 1341 (1989).

12. E. Bulska, B. Godlewska, K. Wróbel and A. Hulanicki, Poster P-E 8, XXVII CSI Post-Symposium, "Speciation of Elements in Environmental and Biological Sciences", Loen, 1991.

13. D. L. Hodges and D. Skelding, Analyst [London], 108, 813 (1983).

14. W. Slavin, J. Anal. At. Spectrosc., 1, 281 (1986).

15. E. Bulska, K. Wróbel and A. Hulanicki, Fresenius' J. Anal. Chem., 342, 740 (1992).

16. E. Bulska, B. Godlewska, K. Wróbel and A. Hulanicki, Can. J. Spectrosc., 36, 89 (1991).

17. A. Hulanicki and E. Bulska, Can. J. Spectrosc., 29, 148 (1984).

(Received November 5, 1991)

(Accepted January 22, 1992) 